DPNU-95-42

hep-ph/9511339

\title{
Dynamical Mass Generation in QED3 with Chern-Simons Term
}

\author{
Pieter MARIS 6 \\ Department of Physics, Nagoya University, Nagoya 464-01, Japan
}

\begin{abstract}
In $(2+1)$-dimensional QED with a Chern-Simons term, we study dynamical breaking of chiral symmetry, using the Dyson-Schwinger equation for the fermions. There is a region in parameter space were dynamical chiral symmetry breaking occurs, just as in pure QED3 (without Chern-Simons term); outside this region, this chiral symmetry breaking solution does not exists. Our results, both numerically and analytically, show that the chiral phase transition is a discontinuous first-order transition.
\end{abstract}

*) E-mail: maris@eken.phys.nagoya-u.ac.jp 


\section{$\S 1$. Introduction}

Dynamical symmetry breaking in (2+1)-dimensional QED has attracted a lot of attention over the last decade (1), (2). From a purely theoretical point of view, it is an interesting and useful theory to study dynamical mass generation. It also has some applications, not only in in elementary particle physics, but also in condensed matter physics, in connection with phenomena occurring in planar surfaces. For particle physics, the main interest in threedimensional theories comes from the fact that they are the high-temperature limit of the corresponding four-dimensional theory, and thus play a role in studies of the early universe and the electroweak phase transition.

A natural extension of pure QED3 is to add a Chern-Simons (CS) term for the gauge field ${ }^{3}$, which breaks parity explicitly. Recently, it has been shown that in the presence of

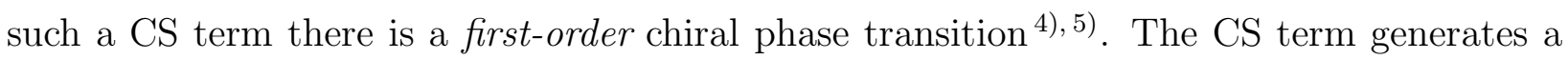
parity-odd mass term for the fermions, but in addition there might be a parity-even mass,

which breaks chirality (6), 团. Both numerical and analytical studies of the truncated DysonSchwinger (DS) equation for the fermion propagator show that there is a first-order phase transition 4 . This is quite remarkable and in contrast to the infinite-order phase transition one finds in pure QED3田, 国)

In this paper, based on Ref.(1) in which more details are given, we show how such a firstorder transition arises, and we also compare some different approximation schemes. In the next section, we introduce the formalism we are using. In Sec. 政 we discuss the DS equation and the truncation schemes we are using, and how this approach leads to dynamical mass generation in pure QED3. Next, we give the results we find in quenched QED3 with CS term. In Sec. 5, we include the effects of the vacuum polarization, and finally we give some concluding remarks in Sec. 6.

\section{$\S 2 . \quad$ Formalism}

The Lagrangian in Euclidean space is

$$
\mathcal{L}=\bar{\psi}\left(i \not \partial+e \not A-m_{e}-\tau m_{o}\right) \psi+\frac{1}{4} F_{\mu \nu}^{2}+\frac{1}{2} i \theta \epsilon_{\mu \nu \rho} A_{\mu} \partial_{\nu} A_{\rho}+\mathcal{L}_{\text {gauge fixing }}
$$

with the dimensionful parameter $\theta$ determining the strength of the CS term. We use fourcomponent spinors for the fermions, and the matrix $\tau$ is defined in such a way that the term $m_{o} \bar{\psi} \tau \psi$ is odd under a parity transformation $\mathrm{G}$, B). Also the CS term is odd under a parity transformation, the other terms in the Lagrangian are parity invariant. 
The inverse full fermion propagator can be written as

$$
S^{-1}(p)=A_{e}(p) \not p+A_{o}(p) \tau \not p-B_{e}(p)-B_{o}(p) \tau .
$$

The functions $A(p)$ and $B(p)$ are scalar functions of $p^{2}$, and their bare values are $A_{e}=1$, $A_{o}=0, B_{e}=m_{e}$, and $B_{o}=m_{o}$. For convenience, we will introduce another decomposition, using the projection operators $\chi_{ \pm} \equiv \frac{1}{2}(1 \pm \tau)$. Defining $A_{ \pm} \equiv A_{e} \pm A_{o}$ and $B_{ \pm} \equiv B_{e} \pm B_{o}$, this leads to

$$
S(p)=-\frac{A_{+}(p) \not p+B_{+}(p)}{A_{+}^{2}(p) p^{2}+B_{+}^{2}(p)} \chi_{+}-\frac{A_{-}(p) \not p+B_{-}(p)}{A_{-}^{2}(p) p^{2}+B_{-}^{2}(p)} \chi_{-} .
$$

With a four-dimensional representation for the $\gamma$-matrices, we can define chirality similar as in four-dimensional QED. Without an explicit mass $m_{e}$ for the fermions, the Lagrangian is chirally symmetric, but the parity-even mass $m_{e}$ breaks this symmetry. Note that the other mass, $m_{o}$, is chirally invariant. Just as in pure QED, the chiral symmetry can be broken dynamically due to nonperturbative effects: starting without a mass term for the fermions in the Lagrangian, there might be a mass generated by the dynamics. This can be studied using the fermion DS equation with the explicit mass $m_{e}$ equal to zero. We also set the bare parity-odd mass, $m_{o}$, equal to zero.

The gauge boson has not only a transverse and longitudinal part, but also a parity-odd part, proportional to $\epsilon_{\mu \nu \rho} q_{\rho}$, due to the explicit CS term in the Lagrangian. In a general covariant gauge, the full gauge boson propagator can be written as

$$
\begin{aligned}
& D_{\mu \nu}(q)=D^{T}\left(q^{2}\right)\left(\delta_{\mu \nu}-\frac{q_{\mu} q_{\nu}}{q^{2}}\right)+D^{O}\left(q^{2}\right) \epsilon_{\mu \nu \rho} \frac{q_{\rho}}{|q|}+a \frac{q_{\mu} q_{\nu}}{q^{4}}, \\
& D^{T}\left(q^{2}\right)=\frac{q^{2}-\Pi^{T}(q)}{\left(q^{2}-\Pi^{T}(q)\right)^{2}+\left(\Pi^{O}(q)-\theta|q|\right)^{2}}, \\
& D^{O}\left(q^{2}\right)=\frac{\Pi^{O}(q)-\theta|q|}{\left(q^{2}-\Pi^{T}(q)\right)^{2}+\left(\Pi^{O}(q)-\theta|q|\right)^{2}},
\end{aligned}
$$

where $\Pi^{T}$ and $\Pi^{O}$ are the transverse and parity-odd part of the vacuum polarization $\Pi_{\mu \nu}$. These parts can be projected out, and this leads to

$$
\begin{aligned}
\Pi^{T}(q)= & -2 N e^{2} \int \frac{\mathrm{d}^{3} k}{(2 \pi)^{3}}\left(k^{2}-2 k \cdot q-3(k \cdot q)^{2} / q^{2}\right) \times \\
& \left\{\frac{A_{+}(k+q) A_{+}(k)}{\left((k+q)^{2} A_{+}^{2}+B_{+}^{2}\right)\left(k^{2} A_{+}^{2}+B_{+}^{2}\right)}+\left[A_{+}, B_{+} \longrightarrow A_{-}, B_{-}\right]\right\}, \\
\Pi^{O}(q)= & \frac{4 N e^{2}}{|q|} \int \frac{\mathrm{d}^{3} k}{(2 \pi)^{3}} q \cdot(k+q) \times \\
& \left\{\frac{A_{+}(k+q) B_{+}(k)}{\left((k+q)^{2} A_{+}^{2}+B_{+}^{2}\right)\left(k^{2} A_{+}^{2}+B_{+}^{2}\right)}-\left[A_{+}, B_{+} \longrightarrow A_{-}, B_{-}\right]\right\},
\end{aligned}
$$


using a bare vertex. Note that the longitudinal part of the vacuum polarization is zero because of gauge invariance.

\section{§3. Dynamical Mass Generation}

In order to study dynamical mass generation for the fermions, we use a truncation of the DS equation for the fermion propagator.

\subsection{Dyson-Schwinger Equation}

The set of DS equations forms a nonperturbative, exact set of equations for Green's functions, and for the fermion propagator it reads

$$
S^{-1}(p)=S_{0}^{-1}(p)-e^{2} \int \frac{\mathrm{d}^{3} k}{(2 \pi)^{3}} \gamma_{\mu} S(k) \Gamma_{\nu}(p, k) D_{\mu \nu}(p-k) .
$$

The problem in solving this equation is the the unknown full vertex function $\Gamma_{\nu}(p, k)$, and the vacuum polarization $\Pi_{\mu \nu}(q)$ which appears in the gauge boson propagator. In principle, we could write down DS equations for these Green's functions as well, but the DS equation for the vertex involves a four-point Green's function, and so on. For practical purposes, we have to truncate the set of DS equations.

In this paper, we consider some different truncation schemes. First, we take the most simple truncation, namely replacing the full vertex and the full gauge boson by the bare ones, see Sec. 1. This is usually referred to as the quenched ladder approximation. We also consider a truncation based on the $1 / N$ expansion.

\section{2. $1 / N$ Expansion}

A popular approximation scheme in QED3 with $N$ fermion flavors is the $1 / N$ expansion 9 ). The coupling constant $e^{2}$ has the dimension of mass, and we can use the large $N$ limit in such a way that $e^{2} \downarrow 0$ and the product $N e^{2}$ remains fixed: $N e^{2}=8 \alpha$ with $\alpha$ fixed. In QED3, the most commonly used truncation scheme of the DS equation is based on this $1 / N$ expansion.

In this truncation scheme, the one-loop vacuum polarization has to be taken into account, since it is of order one: there are $N$ fermion loops, and each loop is proportional to $1 / N$. The full vertex is replaced by the bare one, because that is the leading order contribution in $1 / N$; all vertex corrections are of down by a factor of $1 / N$. This truncation scheme gives two sets of two coupled integral equations for $A_{+}$and $B_{+}$, respectively for $A_{-}$and $B_{-}$

$$
A_{ \pm}(p)=1+\frac{e^{2}}{p^{2}} \int \frac{\mathrm{d}^{3} k}{(2 \pi)^{3}} \frac{2}{k^{2} A_{ \pm}^{2}(k)+B_{ \pm}^{2}(k)} \times
$$




$$
\begin{aligned}
& \left(A_{ \pm}(k)\left(\left(D^{T}(q)-\frac{a}{q^{2}}\right) \frac{q \cdot k q \cdot p}{q^{2}}+\frac{a k \cdot p}{2 q^{2}}\right) \pm B_{ \pm}(k) D^{O}(q) \frac{p \cdot q}{|q|}\right) \\
B_{ \pm}(p)= & e^{2} \int \frac{\mathrm{d}^{3} k}{(2 \pi)^{3}} \frac{1}{k^{2} A_{ \pm}^{2}(k)+B_{ \pm}^{2}(k)} \times \\
& \left(B_{ \pm}(k)\left(2 D^{T}(q)+\frac{a}{q^{2}}\right) \mp 2 A_{ \pm}(k) D^{O}(q) \frac{k \cdot q}{|q|}\right) .
\end{aligned}
$$

where $D^{T}$ and $D^{O}$ are given by Eqs. (5) and (6); $a$ is the gauge parameter. Note that the equations for $A_{+}$and $B_{+}$are coupled to the ones for $A_{-}$and $B_{-}$through the vacuum polarization only, at least in this truncation scheme. The quenched ladder approximation can be recovered by taking a bare gauge boson propagator.

Of course, we have to make sure that we satisfy at least approximately the WardTakahashi identity. One of the consequences of the Ward-Takahashi identity is that the vertex renormalization and the wave-function renormalization have to be equal. This means that if we are using a bare vertex approximation, we have to make sure that $A_{+}=A_{-}=1$, at least approximately. To achieve this, we can use the argument that (formally) the wavefunction renormalization is $1+\mathcal{O}(1 / N)$, see Eq. (10) with $e^{2}=8 \alpha / N$, so to leading order in $1 / N$ we can neglect the wave-function renormalization. Alternatively, we could use a suitable nonlocal (momentum dependent) gauge function $a(q)$, which makes the wave-function renormalization equal to one 10 .

\subsection{Pure QED3}

In QED3 without CS term, dynamical breaking of chiral symmetry has been studied extensively (1), 目). It is known that parity is not broken dynamically ${ }^{1}$ ), so the parity-odd functions $A_{o}$ and $B_{o}$ are zero. Using either a nonlocal gauge such that $A(p)=1$, or just neglecting the wave-function renormalization, the set of Eqs. (10) and (11) reduces to one equation for the dynamical mass function $m(p)=B(p) / A(p)$

$$
m(p)=\frac{8 \alpha}{N} \int \frac{\mathrm{d}^{3} k}{(2 \pi)^{3}} \frac{m(k)}{k^{2}+m^{2}(k)}\left(2 D^{T}(q)+\frac{a}{q^{2}}\right) .
$$

With the one-loop vacuum polarization of bare massless fermions, $\Pi^{T}=-\alpha q$, there exists a critical number of fermion flavors, $N_{c}$, above which there is no chiral symmetry breaking; for $N \leq N_{c}$, there is a dynamically generated fermion mass 1 , Q ). Close to the critical number, the value of the mass function at the origin behaves like

$$
m(0) \sim \alpha \exp \left(\frac{-2 \pi}{\sqrt{N_{c} / N-1}}\right)
$$


corresponding to an infinite-order phase transition. This behavior is obtained both numerically and analytically, using bifurcation theory. Recently, this critical behavior has been confirmed by numerical calculations including the effects of the generated fermion mass on the vacuum polarization 122). The critical number $N_{c}$ depends on the truncation and gauge: in the Landau gauge, $N_{c}=32 / \pi^{2}$, whereas in the nonlocal gauge, one finds $N_{c}=128 /\left(3 \pi^{2}\right)$.

Apart from this difference of a factor of $\frac{4}{3}$, the results in Landau and nonlocal gauge agree with other.

\section{§4. Results with a Chern-Simons Term in Quenched QED}

One important difference with pure QED, is that with a CS term, there is no trivial solution $B_{ \pm}=0$; there is always the chirally-symmetric, parity-odd solution $B_{+}(p)=-B_{-}(p)=$ $B_{o}(p)$, with $B_{e}(p)=0$. This solution can also be found using perturbation theory. Dynamical chiral symmetry breaking can only occur if there is a solution with $B_{+} \neq-B_{-}$, in such a way that $B_{e}(p)=\frac{1}{2}\left(B_{+}(p)+B_{-}(p)\right)$ is nonzero. Furthermore, in the ultraviolet region, the CS term dominates, leading to a behavior of the mass function at large momenta like $B_{ \pm}(p) \sim \pm \theta / p$, whereas the dynamical mass function in pure QED3 falls off like $B(p) \sim 1 / p^{2}$.

\subsection{Numerical Results}

In quenched QED3 with a CS term, the equations for $A_{+}$and $B_{+}$decouple from the equations for $A_{-}$and $B_{-}$, as can be seen from Eqs. (10) and (11) with a bare gauge boson. By choosing the Landau gauge, $a=0$, we find that $A=1$ exactly in pure QED, and for small values of $\theta$ (small compared to the mass scale $e^{2}$ ), $A_{ \pm} \simeq 1$, so we neglect the wave-function renormalization, and consider the equations for $B_{+}$and $B_{-}$only.

Numerically, we find the following solutions:

- $B_{+}(p)=-B_{-}(p)$, the symmetric solution with $B_{e}(p)=0$;

- $B_{+}(0) \simeq \tilde{B}_{-}(0)>0$, with $B_{e}(p) \neq 0$ and $B_{o}(p)=\mathcal{O}(\theta)$.

The last solution exists for small values of $\theta$ only, and corresponds to dynamical breaking of chiral symmetry. For large values of $\theta$, we only find the chirally-symmetric solution. Between these two regions there is a critical value of $\theta$ for dynamical chiral symmetry breaking. As can be seen from Fig. 1, the second solution, $\tilde{B}$, decreases for increasing $\theta$, and beyond some critical value of $\theta$ we cannot find this solution any longer, but at this critical value, $\tilde{B}(0)$ nor the chiral condensate go to zero. This clearly indicates that it is a discontinuous, first-order phase transition. 


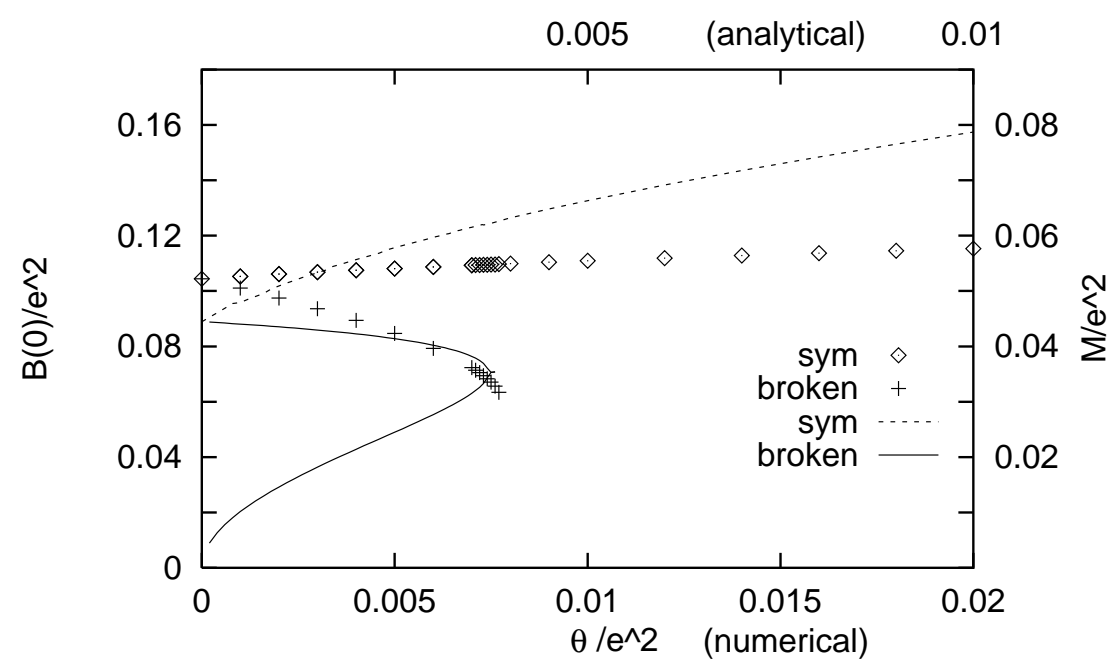

Fig. 1. $B(0)$ versus $\theta$, both numerically (diamonds: symmetric solution, and plusses: broken solution) and analytically (dashed: symmetric, and solid line: broken solution).

\subsection{Analytical Results}

Although the numerical results indicate that it is a first-order phase transition, it is very difficult to establish such a discontinuous transition numerically. In order to solve the equations analytically, we make an expansion in $\theta$, keeping the linear terms only, and perform the angular integrals analytically. Next, we use an Ansatz for the solution based on the leading ultraviolet behavior of the mass functions $B_{ \pm}(p) \simeq \pm e^{2} \theta /\left(\pi^{2} p\right)$, but with a finite value at the origin. Qualitatively, this Ansatz behaves quite similar to the numerical solution we have found in the previous section. This leads to a boundary condition for $M \equiv B_{+}(0)$

$$
M^{3}-\frac{e^{2}}{\pi^{2}}\left(\frac{M^{3}}{|M|}\left(\frac{\pi}{4}-\frac{1}{2} \ln 2\right)+\frac{e^{2} \theta}{2 \pi^{2}}(1+\ln 2)+\frac{\pi \theta M}{2}\right)=0 .
$$

This cubic equation in $M$ has either one or three solutions for given $\theta$, as can be seen from Fig. 2: for small (nonzero) values of $\theta$ there are three solutions, and there is dynamical breaking of chiral symmetry, whereas for large values of $\theta$ there is only the chirally symmetric solution. From this figure it is clear that it is indeed a discontinuous transition, at the critical coupling $\theta_{c}$ the "second" solution $\tilde{B}$ does not go to zero, nor does the chiral condensate. So both our numerical and analytical calculations are in good qualitative agreement, and show that there is a first-order chiral phase

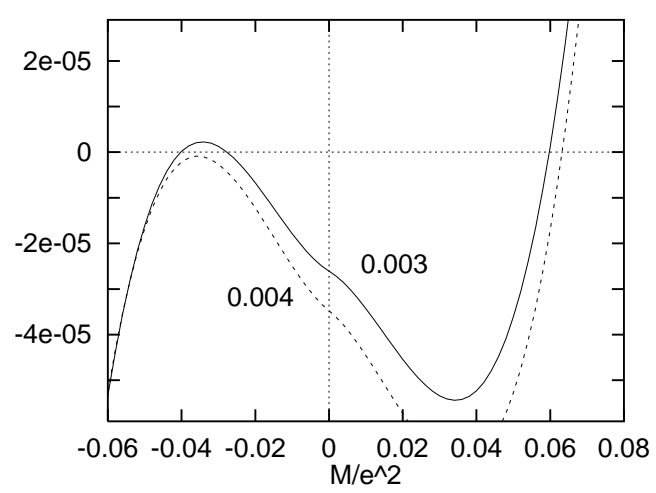

Fig. 2. Boundary condition for $M$ at $\theta=0.003$ and $\theta=0.004$, just below and above $\theta_{c}$. 
transition. Quantitatively there is about a factor of two difference between our numerical and analytical results

$$
\begin{aligned}
\theta_{c} / e^{2} & =0.00371 \text { (an.) } & \theta_{c} / e^{2} \simeq 0.008 \text { (num.) } \\
\tilde{B}_{c} / e^{2} & =0.0352 & \tilde{B}_{c} / e^{2} \simeq 0.06
\end{aligned}
$$

caused by the approximations we made in solving the equation for $B_{ \pm}$analytically.

\section{$\S 5$. Influence of the Vacuum Polarization}

Next, we consider the case of $N$ fermion flavors, and include the effects of the vacuum polarization. Since we know already that there is a critical number of fermion flavors for dynamical chiral symmetry breaking in pure QED, we expect the same to be true in the

presence of a (small) CS term. We first use the one-loop vacuum polarization with bare massless fermions.

\subsection{One-loop Vacuum Polarization}

With massless fermions, there is no parity-odd part of the vacuum polarization, as can be seen from Eq. (8), and the transverse part is simply $\Pi_{T}(q)=-\alpha q$, with $\alpha=N e^{2} / 8$. Thus the transverse and parity-odd part of the gauge boson become

$$
D^{T}(q)=\frac{q^{2}+\alpha q}{q^{2}\left((q+\alpha)^{2}+\theta^{2}\right)}, \quad D^{O}(q)=\frac{-\theta q}{q^{2}\left((q+\alpha)^{2}+\theta^{2}\right)}
$$

Again, the equation for $A_{+}$and $B_{+}$decouple from the ones for $A_{-}$and $B_{-}$, and it should be noted that if we have a solution $\left(A_{+}, B_{+}\right)$of the "plus" equations, then the combination $\left(A_{+},-B_{+}\right)$is automatically a solution of the "minus" equations.

We used both the Landau gauge and a nonlocal gauge $\mathbb{\theta}$ to keep $A_{ \pm} \simeq 1$. In Landau gauge, $A_{ \pm}$will in general not be equal to one, but we nevertheless set it equal to one, in order to be consistent with the bare vertex approximation. Most of the results we present here, are obtained in the nonlocal gauge; in Landau gauge the results are quite similar, the main difference being a scaling factor of $\frac{4}{3}$ in $N$, the number of flavors.

Numerically, we have found results similar to the quenched case, at least below $N_{c}(\theta=0)$, the critical number of fermion flavors in pure QED. For fixed $\theta$, we find a critical $N_{c}(\theta)$, above which there is no chiral symmetry breaking, and also for small $N$, there is a critical $\theta_{c}(N)$ for chiral symmetry breaking. Our numerical results indicate a discontinuous (first-order) phase transition, as can be seen from Fig. 3(a). 

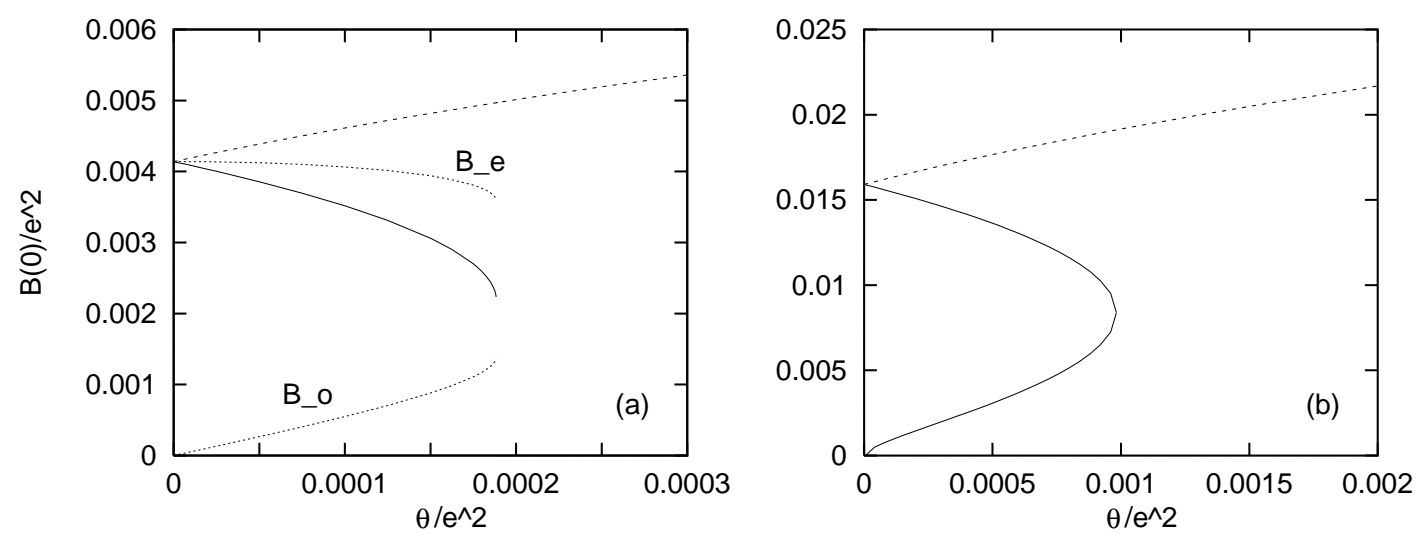

Fig. 3. $B(0)$ (dashed line) and $\tilde{B}(0)$ (solid line) vs. $\theta$ at $N=2$ in nonlocal gauge: (a) numerically and (b) analytically. For completeness, we also included $B_{e}(0)$ and $B_{o}(0)$ in (a).

We also solved the equation analytically, in order to show that the phase transition is indeed first order. Some standard approximations lead to the general solution

$$
B_{ \pm}(p)=M_{ \pm 2} F_{1}\left(a_{+}, a_{-}, \frac{3}{2} ;-p^{2} / M_{ \pm}^{2}\right)
$$

with a boundary condition for $M_{ \pm} \equiv B_{ \pm}(0)$

$$
\begin{array}{r} 
\pm \frac{8 \theta}{\pi^{2} N}\left(1+\frac{16}{9 \pi^{2} N}\right)=M_{ \pm}{ }_{2} F_{1}\left(a_{+}, a_{-}, \frac{1}{2} ;-\alpha^{2} / M_{ \pm}^{2}\right) \\
-\frac{8}{3 \pi^{2} N} M_{ \pm 2} F_{1}\left(a_{+}, a_{-}, \frac{3}{2} ;-\alpha^{2} / M_{ \pm}^{2}\right)
\end{array}
$$

with $a_{ \pm}=\frac{1}{4} \pm \frac{1}{4} i \sqrt{\left(N_{c} / N-1\right)}$; the only difference between the Landau and nonlocal gauge is the critical number: $N_{c}=32 / \pi^{2}$ in Landau and $128 /\left(3 \pi^{2}\right)$ in nonlocal gauge.

This boundary condition gives us $M$ as function of $N$ and $\theta$, and is plotted in Fig. 3(b). As can be seen from this figure, there is a critical $\theta_{c}$, beyond which the chirally symmetric solution is the only one. The generated mass $\tilde{B}$ does not go to zero at the critical point, corresponding to a first-order phase transition. Also in the other direction, increasing $N$ at fixed $\theta$, we find a discontinuous transition, both numerically and analytically ${ }^{4}$. So both our numerical and analytical results are in qualitative agreement with each other; also both Landau and nonlocal gauge are in good qualitative agreement.

In Fig. 4 we have shown the critical line in the $(N, \theta)$ plane.

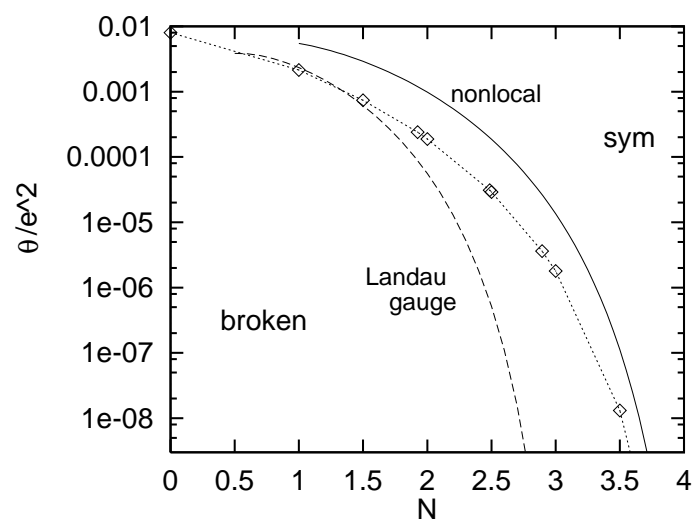

Fig. 4. Phase diagram; the diamonds are numerical results in nonlocal gauge. 


\subsection{Full Vacuum Polarization}

The above results are obtained by using the one-loop vacuum polarization with massless fermions. Now one can of course raise the question what is the influence of the generated mass on the vacuum polarization. In pure QED3, the generated mass has no effect on the critical number of fermion flavors, nor on the behavior of $m(0)$ near the phase transition 12 ), but it does have a crucial effect on the confining properties of this model 13 ) and on behavior of the mass function in the complex momentum plane 44 .

Using the vacuum polarization with the dynamical fermion mass, we have both a transverse and a parity-odd part of the vacuum polarization, and the equations for the "plus" and "minus" functions become coupled. For simplicity, we use the Landau gauge, and put $A_{ \pm}=1$ by hand, so we have to deal with a set of four coupled nonlinear integral equations for $B_{+}, B_{-}, \Pi^{T}, \Pi^{O}$. Our numerical results show that there is dynamical chiral symmetry breaking at small values of $\theta$ and $N$, just as in the previous case. For large values of $\theta$ and $N$, we only find the chirally symmetric solution. In Fig. 5 we have shown our first results. Sofar, the numerical results indicate that the phase transition is again discontinuous, but we need more accurate results close to the critical parameters before we can establish a first-order phase transition; it might be a very sharp second order transition. More detailed calculations of the behavior near the critical line should be done, both numerically and analytically.
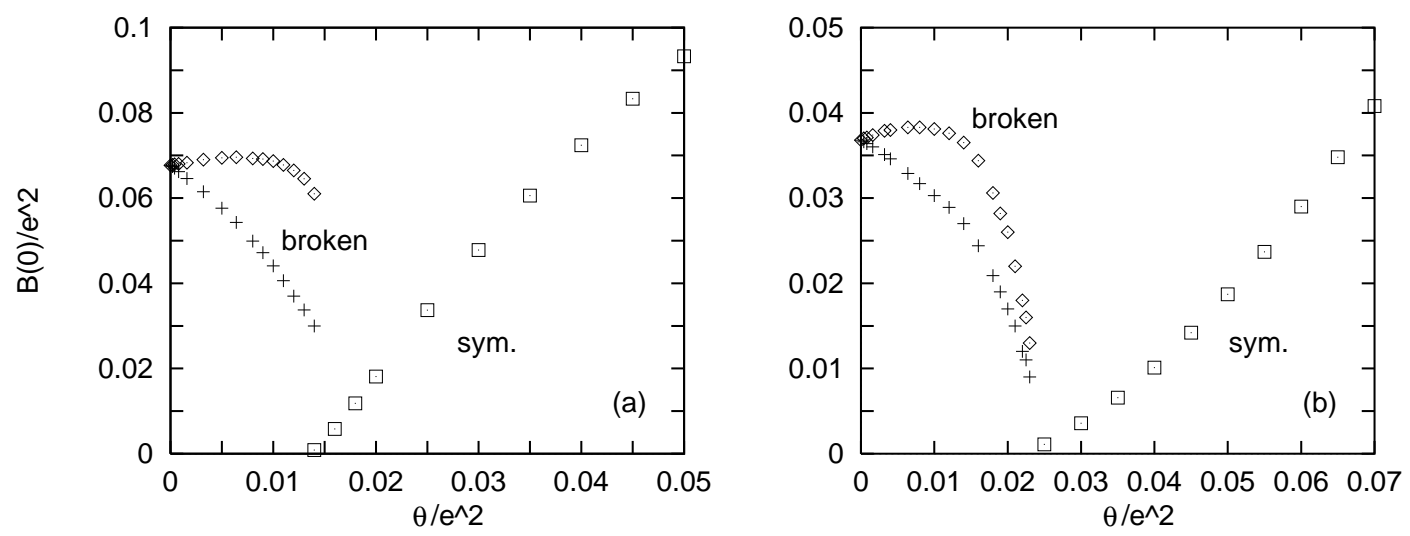

Fig. 5. $\tilde{B}_{+}(0)$ (diamonds), $\tilde{B}_{-}(0)$ (plusses), and $B_{+}(0)$ (squares) vs. $\theta$ at (a) $N=0.5$ and (b) $N=1$.

Finally, we should note that an important feature of vacuum polarization is that the parity-odd part is linear in the generated fermion mass $B$. This linearity turns out to be important: because of this, the symmetric solution, which is parity-odd, vanishes for vanishing $\theta$. This is in agreement with other analyses, showing that there is no dynamical breaking of parity in pure QED311, but in contrast to the results obtained with the one-loop vacuum polarization of massless fermions, see Fig. 3. 


\section{$\S 6$. Conclusion}

In QED3 with $N$ fermion flavors, we have solved the DS equation in the presence of an explicit CS term. Without this CS term, there is dynamical chiral symmetry breaking if the number of fermion flavors is below some critical number $N_{c}$. The chiral phase transition at $N=N_{c}$ is of infinite order; the order parameter and the generated mass vanish continuously at the phase transition.

In the presence of an explicit CS term for the gauge field, there can be both a chirally symmetric mass, and a mass which breaks the chiral symmetry. Below the critical number $N_{c}$, we find that there is chiral symmetry breaking for small values of $\theta$, just as in pure QED3. There is a critical line in the $(N, \theta)$ plane, beyond which the chirally symmetric solution is the only solution. At this critical line, the order parameter and the generated mass do not vanish, corresponding to a first-order phase transition. Both numerically and analytically we have found this discontinuous transition both in quenched QED3, and using the one-loop vacuum polarization. First results including the generated mass in the vacuum polarization indicate the same behavior.

Note that there is no dynamical breaking of parity. In our analysis, this can be seen from the fact that the chirally-symmetric, parity-odd solution vanishes for vanishing $\theta$. One has to take into account the effect of the generated fermion mass on the vacuum polarization in order to get this result.

\section{Acknowledgements}

Most of this work has been done in collaboration with K.-I. Kondo. I would also like to thank Y. Kim, K. Yamawaki and D.K. Hong for interesting discussions. This work is financially supported by the Japanese Society for the Promotion of Science.

\section{References}

[1] R.D. Pisarski, Phys. Rev. D29 (1984), 2423; T.W. Appelquist, M.J. Bowick, D. Karabali, and L.C.R. Wijewardhana, Phys. Rev. D33 (1986), 3704; T.W. Appelquist, D. Nash, and L.C.R. Wijewardhana, Phys. Rev. Lett. 60 (1988), 2575; D. Nash, Phys. Rev. Lett. 62 (1989), 3024.

[2] E. Dagotto, A. Kocić, and J.B. Kogut, Phys. Rev. Lett. 62 (1989), 1083; Nucl. Phys. B334 (1990), 279.

[3] S. Deser, R. Jackiw, and S. Templeton, Ann. Phys. 140 (1982), 372. 
[4] K.-I. Kondo and P. Maris, Phys. Rev. Lett. 74 (1995), 18; Phys. Rev. D52 (1995), 1212.

[5] K.-I. Kondo, First and Second Order Phase Transition in Maxwell-Chern-Simons Theory Coupled to Fermions, CHIBA-EP-89, hep-ph/9509345.

[6] D.K. Hong and S.H. Park, Phys. Rev. D47 (1993), 3651.

[7] K.-I. Kondo, T. Ebihara, T. Iizuka and E. Tanaka, Nucl. Phys. B434 (1995), 85.

[8] G.W. Semenoff and L.C.R. Wijewardhana, Phys. Rev. Lett. 62 (1988), 2633; M. Carena, T.E. Clark, and C.E.M. Wagner, Phys. Lett. B259 (1991), 128; Nucl. Phys. B356 (1991), 117.

[9] R. Jackiw and S. Templeton, Phys. Rev. D23 (1981), 2291; T.W. Appelquist and R.D. Pisarski, Phys. Rev. D23 (1981), 2305; T.W. Appelquist and U. Heinz, Phys. Rev. D23 (1981), 2169.

[10] E.H. Simmons, Phys. Rev. D42 (1990), 2933; T. Kugo and M.G. Mitchard, Phys. Lett. B282 (1992), 162.

[11] T.W. Appelquist, M.J. Bowick, D. Karabali, and L.C.R. Wijewardhana, Phys. Rev. D33 (1986), 3774; C. Vafa and E. Witten, Commun. Math. Phys. 95 (1984), 257.

[12] V.P. Gusynin, A.H. Hams, and M. Reenders, (2+1)-Dimensional QED with Dynamically Massive Fermions in the Vacuum Polarization, UG-10-95, hep-ph/9509380.

[13] C.J. Burden, J. Praschifska, and C.D. Roberts, Phys. Rev. D46 (1992), 2695.

[14] P. Maris, Confinement and Complex Singularities in QED3, DPNU-95-20, hepph/9508323. 